\title{
Comparative analysis of human embryonic stem cell-derived neural stem cells as an in vitro human model
}

\author{
JUNG-HWA OH ${ }^{1,2^{*}}$, CHO-ROK JUNG ${ }^{3,4^{*}}$, MI-OK LEE ${ }^{5}$, JANGHWAN KIM $^{3,4}$ and MI-YOUNG SON ${ }^{3,4}$ \\ ${ }^{1}$ Korea Institute of Toxicology, Daejeon 34114; ${ }^{2}$ Department of Human and Environmental Toxicology, \\ Korea University of Science and Technology, Daejeon 34113; ${ }^{3}$ Stem Cell Research Center, Korea Research Institute of \\ Bioscience and Biotechnology, Daejeon 34141; ${ }^{4}$ Department of Functional Genomics, Korea Research Institute of \\ Bioscience and Biotechnology, School of Bioscience, Korea University of Science and Technology, Daejeon 34113; \\ ${ }^{5}$ Immunotherapy Convergence Research Center, Korea Research Institute of \\ Bioscience and Biotechnology, Daejeon 34141, Republic of Korea
}

Received August 7, 2017; Accepted November 29, 2017

DOI: $10.3892 /$ ijmm.2017.3298

\begin{abstract}
Alternative cell models of human neural stem cells (hNSCs) have been developed and used for investigations ranging from in vitro experiments to in vivo clinical studies. However, a cell model capable of mimicking the 'normal' state of hNSCs is mandatory in order to extrapolate the results of these studies to humans. In the present study, to select a more suitable hNSC model for developing human-based experimental platforms, two representative hNSC types were compared, namely human embryonic stem cell (hESC)-derived hNSCs and ReNcell CX cells, which are well-characterized immortalized hNSC lines. The hNSCs, differentiated from hESCs via human neuroectodermal sphere (hNES) formation, recapitulated the molecular and cellular phenotypes of hNSCs, including NSC marker expression and terminal neuronal differentiation potential. Comparative analyses of the transcriptome profiles of the hESC-derived hNESs and ReNcell CX hNSCs showed that the differentiated hNESs were analogous to the ReNcell CX cells, as demonstrated
\end{abstract}

Correspondence to: Dr Mi-Young Son or Dr Janghwan Kim, Stem Cell Research Center, Korea Research Institute of Bioscience and Biotechnology, 125 Gwahangno, Yuseong-gu, Daejeon 34141, Republic of Korea

E-mail: myson@kribb.re.kr

E-mail: janghwan.kim@kribb.re.kr

${ }^{*}$ Contributed equally

Abbreviations: bFGF, basic fibroblast growth factor; EGF, epidermal growth factor; hEB, human embryoid body; hESC, human embryonic stem cell; hNSC, human neural stem cell; NES, neuroectodermal sphere; NSC, neural stem cell; PCA, principal component analysis

Key words: neural stem cell, neural sphere, human embryonic stem cell, ReNcell, microarray, differentiation by principal component analysis and hierarchical sample clustering. The hNSC-specific transcriptome was presented, comprising commonly expressed transcripts between hNESs derived from hESCs and ReNcell CX cells. To elucidate the molecular mechanisms associated with the hNSC identity, the hNSC-specific transcriptome was analyzed using pathway and functional annotation clustering analyses. The results suggested that hESC-derived hNESs, an expandable and accessible cell source, may be used as a relevant hNSC model in a wide range of neurological investigations.

\section{Introduction}

Over the last two decades, neural stem cells (NSCs) have become a major topic of interest from basic research to translational experiments for the development of therapies for a range of neurological disorders. NSCs have two defining characteristics: Self-renewal and multipotentiality (1). Their capacity to propagate in culture over several passages and differentiate into neuronal and glial cell types renders them attractive as a model of neurogenesis and neural cells, and as a therapeutic tool for treating neurological disease. Previous studies have used a wide range of NSCs from adult and fetal origins, but predominantly from rodent models (2). However, in the case of human NSCs (hNSCs), several constraints, including the limited donor availability to derive fetal and adult NSCs, the low rate of proliferation and the difficulty of long-term in vitro expansion, mean it is not possible to produce the required cell numbers while maintaining a stable phenotype across passages. Therefore, it is important to develop in vitro expandable cell sources for providing suitable hNSCs in sufficiently large numbers.

The life span of hNSCs in vitro can be improved by optimizing culture conditions (3) or via immortalization using the myc transcription factor (4) and maintaining a stable phenotype. Stable hNSC lines, including ReNcell CX cells immortalized using c-myc and VM cells immortalized with v-myc, are widely used in investigations in a variety of neurological fields (5). ReNcell lines have been shown to 
propagate perpetually in culture and exhibit properties of hNSCs, including expression of NESTIN in an undifferentiated state and differentiation into specific cell types, including neuronal and glial cells, following deprivation of growth factors in culture medium (6). It was previously reported that ReNcell lines were used in disease modeling for Alzheimer's disease (AD) (7,8); a three-dimensional culture model of ReNcell VM cells with mutations in amyloid precursor protein and presenilin 1 was able to recapitulate AD pathologies. However, there are practical limitations to using immortalized hNSC lines for clinical applications, including a higher risk of aberrant growth, which may be circumvented by subjecting these cells to extensive characteristic analyses.

Human embryonic stem cells (hESCs), used as pluripotent cells, provide an unlimited and renewable source of hNSCs. Several protocols have been developed to differentiate hESCs into expandable hNSC populations, and to derive potentially functional neurons and glial cells in a controlled manner $(6,9,10)$. Due to the high differentiation potential, in vitro expandable NSCs derived from hESCs are one of the most accessible models for human developmental neurobiology, although certain ethical issues remain unresolved (11). hESC-derived NSCs can serve as an in vitro model for the examination of human neural development as newly derived NSCs are similar to embryonic neuroepithelial cells. In addition, in long-term culture, these cells are more likely to develop features similar to those of fetal and adult NSCs (12). The hESCs used in the production of hNSCs have the advantage of being capable of propagation over multiple passages, offering a virtually unlimited supply of hNSCs (13).

The present study aimed to compare and characterize two representative hNSC sources to provide a well-defined in vitro model comparable to human neuronal physiology for various research applications. This involved examining whole-genome expression using microarrays in ReNcell and hESC-derived NSCs, and assessing their neuronal differentiation potential. To the best of our knowledge, this is the first report to provide a comprehensive analysis of the gene expression of ReNcell and hESC-derived NSCs. The results extend the gene expression network for neural differentiation and reveal common principles of transcriptional regulation underlying the differentiation of hESCs into NSCs.

\section{Materials and methods}

hESC culture. H9 hESCs (cat. no. WA09; WiCell Research Institute, Madison, WI, USA) were maintained on Matrigel (BD Biosciences, San Diego, CA, USA) in mTeSR1 (StemCell Technologies, Vancouver, BC, Canada) as previously described $(14,15)$.

Differentiation of hESCs into hNSCs. The hNSCs were differentiated through the formation of human neuroectodermal spheres (hNESs) as previously reported with minor modifications $(2,16)$. The H9 hESCs (cat. no. WA09; WiCell Research Institute) were maintained on Matrigel (BD Biosciences) in mTeSR1 (StemCell Technologies) as previously described (14). Human embryoid bodies (hEBs) were generated by culturing hESCs in hEB medium consisting of knockout DMEM supplemented with $10 \%$ knockout serum replacement,
$1 \%$ non-essential amino acids, $1 \mathrm{mM}$ L-glutamine (all from Invitrogen; Thermo Fisher Scientific, Inc., Waltham, MA, USA) and $0.1 \mathrm{mM} \beta$-mercaptoethanol (Sigma-Aldrich; Merck KGaA, Darmstadt, Germany) on non-coated Petri dishes. The resulting hEBs were then cultured in NES/NSC medium consisting of DMEM/F12, 1X N2/B27 (both from Invitrogen; Thermo Fisher Scientific, Inc.), $20 \mathrm{ng} / \mathrm{ml}$ basic fibroblast growth factor (bFGF; R\&D Systems, Inc., Minneapolis, MN, USA), $20 \mathrm{ng} / \mathrm{ml}$ epidermal growth factor (EGF) and $10 \mathrm{ng} / \mathrm{ml}$ leukemia inhibitory factor (both from PeproTech, Inc., Rocky Hill, NJ, USA). The hNESs were sub-cultured every week using a Mcllwain tissue chopper (Mickle Engineering, Surrey, UK), and the medium was replaced every 2 days. The hNESs were passaged at least five times without disturbing the formation of neural rosettes. For terminal differentiation, each hNES was allowed to attach to a Matrigel-coated coverslip and was maintained without growth factors for 2 weeks, as previously described $(17,18)$. To count the total number of cells within each hNES, the hNESs were dissociated into single-cell suspensions with $0.1 \%$ trypsin-EDTA (Invitrogen; Thermo Fisher Scientific, Inc.) for $3 \mathrm{~min}$. Live cell numbers were counted using trypan blue (Invitrogen; Thermo Fisher Scientific, Inc.) exclusion under an Olympus fluorescence microscope (IX51; Olympus Corp., Tokyo, Japan).

ReNcell CX cell culture. ReNcell CX cells derived from the cortical region of human fetal brain tissue (cat. no. SCC007; EMD Millipore, Temecula, CA, USA) were cultured according to the manufacturer's protocol. The ReNcell CX cells were maintained in ReNcell NSC maintenance medium supplemented with $20 \mathrm{ng} / \mathrm{ml}$ EGF and $20 \mathrm{ng} / \mathrm{ml} \mathrm{bFGF} \mathrm{(all}$ from EMD Millipore) on laminin-coated tissue culture dishes (BD Biosciences). The culture medium was replaced every 2 days. For terminal differentiation, the ReNcell CX cells were cultured for 5 days without growth factors, as previously described (19).

Immunocytochemistry. Immunocytochemistry was performed as previously described (20). In brief, the cells were fixed in $4 \%$ formaldehyde and then permeabilized with PBS containing $0.1 \%$ Triton $\mathrm{X}-100$. Following blocking with $3 \%$ bovine serum albumin (Sigma-Aldrich; Merck KGaA), the cells were incubated at $4^{\circ} \mathrm{C}$ overnight with anti-neuron-specific class III $\beta$-tubulin (TUJ1; 1:500; cat. no. PRB-435P; Covance, Inc., Princeton, NJ, USA), anti-NESTIN (1:100; cat. no. MAB5326), anti-microtubule-associated protein 2 (MAP2; 1:500; cat. no. MAB3418), anti-glial fibrillary acidic protein (GFAP; 1:200; cat. no. MAB3402) and anti-Ki67 (1:500; cat. no. AB9260; Chemicon) (all from EMD Millipore), followed by incubation with Alexa Fluor 488-conjugated anti-mouse IgG (1:1,000; cat. no. A21202), Alexa Fluor 594-conjugated anti-mouse IgG (1:1,000; cat. no. A21203), Alexa Fluor 488-conjugated anti-rabbit IgG (1:1,000; cat. no. A21441) or Alexa Fluor 594-conjugated anti-rabbit IgG (1:1,000; cat. no. A21442) (all from Molecular Probes, Eugene, OR, USA) as secondary antibodies for $1 \mathrm{~h}$ at room temperature. DAPI (1 mg/ml; Invitrogen; Thermo Fisher Scientific, Inc.) was added to visualize the nuclei. The slides were examined using an Axiovert 200M microscope (Carl Zeiss AG, Gottingen, Germany). 
Table I. List of primers used in the present study.

\begin{tabular}{|c|c|c|}
\hline Gene & Forward primer $\left(5^{\prime}-3^{\prime}\right)$ & Reverse primer $\left(5^{\prime}-3^{\prime}\right)$ \\
\hline OCT4 & GAGAAGGATGTGGTCCGAGTGTG & CAGAGGAAAGGACACTGGTCCC \\
\hline SOX2 & AGAACCCCAAGATGCACAAC & ATGTAGGTCTGCGAGCTGGT \\
\hline SOX1 & GGGAAAACGGGCAAAATAAT & CCATCTGGGCTTCAAGTGTT \\
\hline SOX3 & GACGCCTTGTTTAGCTTTGC & TTCTCССАTТСАСТССТTGG \\
\hline MSII & ACССССАСАТТСТСТСАСТG & AAACCCAAAACACGAACAGC \\
\hline TUJ1 & ACCTCAACCACCTGGTATCG & GGGTACCACTCCACGAAGTA \\
\hline NESTIN & CAGGAGAAACAGGGCCTACA & TGGGAGCAAAGATCCAAGAC \\
\hline GAPDH & GAAGGTGAAGGTCGGAGTC & GAAGATGGTGATGGGATTTC \\
\hline
\end{tabular}

OCT4, octamer-binding protein 4; SOX, superoxide dismutase; MSI1, musashi-1; TUJ1, neuron-specific class III $\beta$-tubulin.

Semi-quantitative reverse transcription-polymerase chain reaction (RT-PCR) analysis. Total RNA was extracted from cells with an RNeasy kit (Qiagen, Inc. Hilden, Germany) and reverse transcribed using a Superscript IV First-Strand Synthesis System kit (Invitrogen; Thermo Fisher Scientific, Inc.) as previously described (21). The resulting cDNA was diluted 1:10 with deionized water, and $1 \mu \mathrm{l}$ of the diluted cDNA was added to Accupower ${ }^{\mathrm{TM}}$ PCR PreMix (Bioneer Corp., Daejeon, Korea), 10 pmol/1 of specific primers and deionized water to a final volume of $20 \mu \mathrm{l}$. The RT-PCR analysis was performed under the following conditions: $5 \mathrm{~min}$ at $95^{\circ} \mathrm{C} ; 30-40$ cycles of $30 \mathrm{sec}$ at $95^{\circ} \mathrm{C}, 30 \mathrm{sec}$ at $60^{\circ} \mathrm{C}, 30 \mathrm{sec}$ at $72^{\circ} \mathrm{C}$, and $5 \mathrm{~min}$ extension at $72^{\circ} \mathrm{C}$. GAPDH was used as an internal control. The relative expression of target genes was determined using the $2^{-\Delta \Delta \mathrm{Cq}}$ method (22). The primers used in this study are listed in Table I.

Microarray analysis. The microarray experiments were performed using the Low RNA input linear amplification kit, cRNA cleanup module and one-color (Cy3) Whole Human Genome Microarray 4X44K, according to the manufacturer's protocol (Agilent Technologies, Inc., Santa Clara, CA, USA) as previously described (23). The raw data was normalized using global scale normalization and processed using GeneSpring software version 11.0 (Agilent Technologies, Inc.,). Heatmap and hierarchical clustering of genes was generated using $\mathrm{MeV}$ v. 4.9.0 software (http://www.tm4.org). Gene functions were annotated using the GeneCard database (http://www.genecards.org/). The principal component analysis (PCA) was performed with GeneSpring software. Biological processes and protein classes were described using Protein Analysis Through Evolutionary Relationships (PANTHER; http://www.pantherdb.org/). Kyoto Encyclopaedia of Genes and Genomes (KEGG) analysis and functional annotation clustering were performed using David Bioinformatics Resources 6.8 with the Database for Annotation, Visualization and Integrated Discovery (DAVID; http://david.abcc.ncifcrf.gov).

\section{Results and discussion}

Generation of NSCs derived from hESCs via hNES formation. In the present study, H9 hESCs were differentiated into hNSCs based on the previously described hNES formation method (17). The aggregates of hESCs were cultured in hEB medium for 5 days, followed by transfer into NES/NSC medium to drive neuronal fate commitment and promote neuronal differentiation (Fig. 1A). During differentiation, following the first subculture, hNESs containing neural rosette structures, a key structure representing NSCs, appeared and retained the potential to form neural rosette structures (Fig. 1A; red dotted circle). The hNESs were dissociated into single NSCs by trypsin digestion and were cultured as adherent monolayers. As reported in our previous study $(1,17,24)$, the hNESs generated using this method are characterized as NSCs as they have the potential to differentiate into neuronal and glial cell types, and can be serially passaged to form new hNESs.

Immortalized hNSC lines are in increasing demand due to the inherent limitations of primary hNSCs, including limited availability, poor expandability and associated ethical issues (5). The ReNcell CX cell line, which is a commercially available immortalized fetal cortical NSC line, was used as a reference hNSC type in the present study. Under normal growth conditions, ReNcell CX cells exhibited immature neural morphology, similar to that of monolayer-cultured hNSCs (Fig. 1). The ReNcell CX cells grew rapidly as a monolayer on laminin, with a doubling time of $\sim 24 \mathrm{~h}$ due to the c-MYC-based immortalization. Therefore, there are safety concerns, including the risk that oncogenic c-MYC may render this hNSC line tumorigenic following transplantation $(19,25)$.

Comparative gene expression analysis of hNESs derived from hESCs and ReNcell CX cells. To compare hNESs derived from hESCs and ReNcell CX cells for use as an hNSC model, and examine the mechanisms underlying lineage commitment in NSCs, microarray analyses were performed in undifferentiated hESCs, hEBs (intermediate cells in hNSC differentiation), hESC-derived hNESs and ReNcell CX cells. A heatmap showing the hierarchical clustering results from the whole-genome expression profiles indicated that differentiated hNESs preferentially clustered with ReNcell CX cells (Fig. 2A). The principal component analysis (PCA) also confirmed that hNESs and ReNcell CX cells were distinctly separated from undifferentiated hESCs and hEBs (Fig. 2B). Accordingly, only $\sim 12.4 \%$ of all the genes were differentially expressed, with a fold-change threshold of 2.0 between the hNESs and ReNcell CX cells. These data indicated that the 


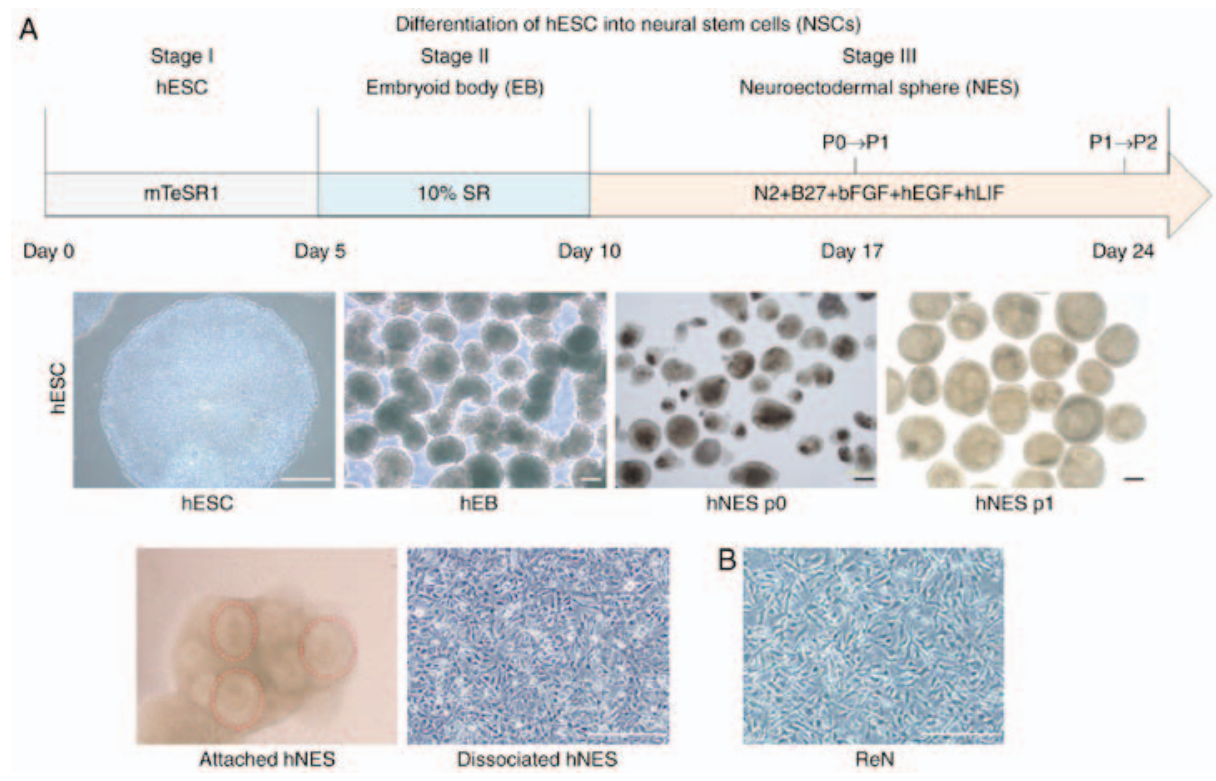

Figure 1. Differentiation of hESCs into NESs containing NSCs. (A) Schematic diagram of the protocol used to obtain NSCs from hESCs via NES formation. Representative cell images are shown for each step. (B) hESCs were allowed to form hEBs, grown for 5 days and then transferred to NES/NSC medium. Following the first passage, rosette-containing NESs appeared. Prominent rosette-like structures were observed in the attached NESs and are indicated by the red dotted circles. NESs were dissociated into single cells and expanded by mechanical passaging. Representative images of ReNcell CX cells are shown. Scale bar $=200 \mu \mathrm{m}$. hESCs, human embryonic stem cells; NES, neuroectodermal sphere; NSC, neural stem cell; hEBs, human embryonic bodies; ReN, ReNcell CX cells.

global transcription of differentiated hNESs is similar to that of ReNcell CX cells.

To obtain the overall profile regarding common aspects of hNSC identity, a total of 1,711 commonly upregulated and 856 commonly downregulated genes between hNESs and ReNcell CX cells with a fold-change of $>5$ were identified as the hNSC-specific transcriptome and analyzed using the PANTHER classification system. The top biological process term was cellular process (26.9\%) (Fig. 2C). Other major processes corresponding to these hNSC-related genes included metabolic process $(20.4 \%)$, response to stimulus $(8.7 \%)$, developmental process (8.3\%), biological regulation (7.6\%), localization (7.3\%), multicellular organismal process $(6.3 \%)$ and cellular component organization or biogenesis (5.6\%), as shown in Fig. 2C. In addition, the predominant protein class was nucleic acid binding (12.9\%), followed by hydrolase (8.9\%), enzyme modulator $(8.6 \%)$, signaling molecule $(8.5 \%)$, transcription factor $(8.3 \%)$, receptor $(7.0 \%)$, transporter $(6.6 \%)$, transferase $(6.5 \%)$ and cytoskeletal protein $(5.6 \%)$, as shown in Fig. 2D. A significant number of genes were identified as transcription factors, which are known to have prominent roles in lineage specification and developmental processes (26). Therefore, the contribution of these transcription factors to the hNSC identity was dissected. The important enriched transcription factor categories were zinc finger transcription factor (38.4\%), helix-turn-helix transcription factor (25.6\%), transcription cofactor (18.4\%), basic helix-loop-helix transcription factor (8.0\%), HMG box transcription factor (4.8\%), and nuclear hormone receptor binding (4.0\%), as shown in Fig. 2D. The expression of several transcription factors from the microarray data were analyzed further, and the transcription factor expression levels were similar between the hNESs and ReNcell CX cells (Fig. 2E). Representative genes are shown in Fig. 3.
Gene ontology $(G O)$ and pathway enrichment analysis in hNESs derived from hESCs and ReNcell CX cells. To elucidate the signaling pathways and molecular mechanisms associated with the hNSC identity, the present study analyzed the hNSC-specific transcriptome. Pathway analysis based on the KEGG database showed that the commonly upregulated genes were significantly associated with the following pathways: Cytokine-cytokine receptor interaction, PI3K-Akt signaling, complement and coagulation cascades, axon guidance, focal adhesion, ECM-receptor interaction, Ras signaling, proteoglycans in cancer, regulation of actin cytoskeleton, and $\mathrm{ABC}$ transporters (Fig. 4A). Cell adhesion molecules, metabolic pathways, antigen processing and presentation, glycerophospholipid metabolism, T cell receptor signaling, cell cycle, oxytocin signaling, biosynthesis of antibiotics, ErbB signaling, and progesterone-mediated oocyte maturation were enriched for the commonly downregulated genes in hNESs and ReNcell CX cells (Fig. 4B).

To obtain a more comprehensive understanding of the functions of the hNSC-specific transcriptome, GO term enrichment analysis was performed through DAVID functional annotation clustering, which shows functionally linked groups by reducing the redundancy in the annotation $(11,27)$. The following seven significant annotation clusters were identified in the hNSC-specific transcriptome, which were related to glycoprotein, cell junction (synapse), immunity, EGF-like domain, ECM-receptor interaction (fibrillar collagen), regulation of PI3K signaling and metal-binding based on statistical criteria $(\mathrm{P}<0.05$ and an enrichment score of at least 1.7) (Fig. 4C).

Although the majority of the genes analyzed showed similar expression patterns, differentially expressed genes found only in a small portion of genes ( $12.4 \%$ of all genes given the 2.0 -fold cutoff criterion) between hNESs and ReNcell CX cells were enriched in the following pathways: TGF- $\beta$ signaling, acute 

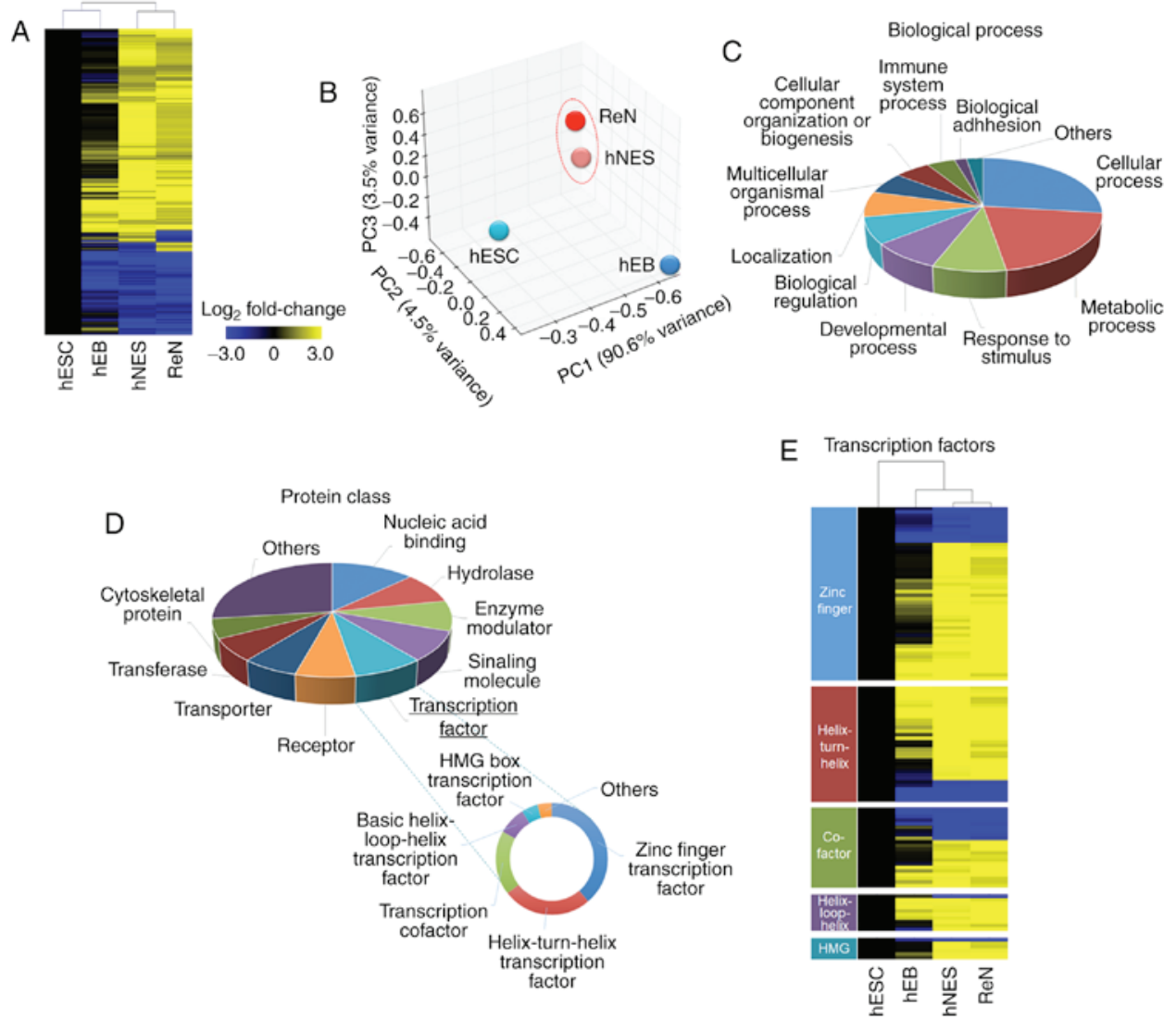

Figure 2. Global analyses of transcriptome changes during NSC differentiation of hESCs. (A) Heatmap of undifferentiated hESCs, hEBs, hESC-derived hNESs and ReNcell CX cells. Genes in which expression was not significantly altered (<2-fold) were removed. (B) Principal component analysis of the differentially expressed genes from the microarray data. (C) Enriched biological processes and (D) protein classes of commonly regulated genes in hNESs and ReNcell CX cells by PANTHER analysis. Genes related to transcription factors were further classified. (E) Heatmaps of genes known to be involved in CM differentiation, including genes associated with transcription factors in hESCs, hEBs, hNESs and ReNcell CX cells. Hierarchical clustering showed a close association between hNESs and ReNcell CX cells. hESCs, human embryonic stem cells; hNESs, human neuroectodermal spheres; hEBs, human embryonic bodies; ReN, ReNcell CX cells.

\begin{tabular}{|c|c|c|c|c|c|}
\hline \multicolumn{6}{|c|}{ Upregulated genes } \\
\hline Gene symbel & hESC & hEB & hNES & $\begin{array}{l}\text { ReNcell } \\
\text { Cx }\end{array}$ & Agilent probe ID \\
\hline SCARF2 & 100 & 1791 & 25579 & 1286.75 & A_23_P132185 \\
\hline CST7 & 100 & 813 & 17872 & 65257 & A_23_P68601 \\
\hline MDGA1 & 100 & 157.20 & 139.69 & 63018 & A_23_P310460 \\
\hline C8ort46 & 100 & 20.99 & 33384 & 44653 & A_23_P353614 \\
\hline CCDC92 & 100 & $\$ .77$ & 177.66 & 266.69 & A_23_P98900 \\
\hline DNHD1 & 100 & 1263 & 17266 & 20731 & A_23_P316472 \\
\hline GLIS3 & 1.00 & 24.33 & 8135 & 17991 & A 32 P39394 \\
\hline KCTD4 & 100 & 8.44 & 148.49 & 174.29 & A_ 23_P 48327 \\
\hline SERPINA3 & 100 & 0.98 & 113.78 & 17157 & A_23_P2920 \\
\hline APC2 & 100 & 26,11 & 11488 & 14222 & A 23 P101908 \\
\hline NDST4 & 1.00 & 4.82 & 11535 & 138.72 & A 23 P 136371 \\
\hline ZFHX4 & 1.00 & 21.77 & 14702 & 125.89 & A 23_P43095 \\
\hline SVEP1 & 100 & 559 & 111.42 & 123.70 & A_23_P216596 \\
\hline MGP & 100 & 1052 & 14354 & 11748 & A_23_P204286 \\
\hline SLCBA1 & 100 & 3.48 & 5927 & 88.66 & A 24_P136866 \\
\hline CYP4F3 & 100 & 4.56 & 8231 & 87.75 & A_23 P209116 \\
\hline C7ort33 & 100 & 13.19 & 6003 & 86.19 & A_23_P405161 \\
\hline CYP11B2 & 100 & 304 & 103 is & 1963 & A 23_P215997 \\
\hline EYA4 & 100 & 251 & 67.23 & 75.45 & A_23_P145507 \\
\hline NEU3 & 100 & 198 & 54.77 & 61.89 & A 23 P203716 \\
\hline LDHALEB & 100 & 271 & 8892 & 59.16 & A_23_P54357 \\
\hline DCTN1 & 100 & 14,39 & 7783 & 5503 & A_24_P917744 \\
\hline CHRM3 & 100 & 0.88 & 5239 & 54.69 & A_24_P25137 \\
\hline CLTB & 1.00 & 2.22 & 56.31 & 53.62 & A_23_P252671 \\
\hline RANBP3L & 100 & 9.11 & 63.33 & 50.90 & A_23_P144929 \\
\hline
\end{tabular}

\begin{tabular}{|c|c|c|c|c|c|}
\hline \multicolumn{6}{|c|}{ Downregulated genes } \\
\hline Gene symbol & hESC & hEB & hNES & $\begin{array}{l}\text { ReNcell } \\
\text { Cx }\end{array}$ & Aglent probe ID \\
\hline ACTA1 & 100 & 0.21 & 0.01 & 0.01 & A 23 P1102 \\
\hline ARHGEF5 & 100 & 0.51 & 0.02 & 0.01 & A 23_P93988 \\
\hline CuzD1 & 100 & 0.06 & 0.01 & 0.01 & A_23_P47058 \\
\hline DPPA4 & 1.00 & 0.08 & 0.02 & 0.01 & A 23 P 380526 \\
\hline FZD5 & 100 & 1.36 & 0.01 & 0.01 & A_23_P108437 \\
\hline GABRB3 & 100 & 0.04 & 0.02 & 0.01 & A 23 P10966 \\
\hline GAL & 100 & 0.12 & 0.02 & 0.01 & A 23_P 374844 \\
\hline L1TD1 & 1.00 & 0.23 & 0.01 & 0.01 & A_23_P137484 \\
\hline PHF8 & 100 & 0.09 & 0.02 & 0.01 & A_24_P130865 \\
\hline POUSF1 & 100 & 0.06 & 0.01 & 0.01 & A_24_P144601 \\
\hline PPAP2C & 1.00 & 0.10 & 0.01 & 0.01 & A 23 P108294 \\
\hline$S C G B 3 A 2$ & 1.00 & 0.11 & 0.01 & 0.01 & A 23 P145006 \\
\hline TDGF3 & 100 & 0.07 & 0.01 & 0.01 & A 24 P179646 \\
\hline VENTX & 100 & 0.11 & 0.01 & 0.01 & A_23_P419150 \\
\hline ZFP42 & 100 & 0.18 & 0.01 & 0.01 & A_23_P395582 \\
\hline OR2A2OP & 100 & 0.13 & 0.02 & 0.01 & A 24_P506816 \\
\hline RAB $11 \mathrm{~B}$ & 100 & 0.01 & 0.02 & 0.01 & A 23 P 67748 \\
\hline HSPA8 & 100 & 0.03 & 0.01 & 0.01 & A_24_P295745 \\
\hline TRIM6 & 1.00 & 0.11 & 0.02 & 0.01 & A_24_P381199 \\
\hline TRPV4 & 100 & 0.15 & 0.02 & 0.01 & A 24 P13381 \\
\hline ATP2B2 & 100 & 0.47 & 0.02 & 0.02 & A 23 P18152 \\
\hline WDR46 & 100 & 0.02 & 0.02 & 0.02 & A_24_P389038 \\
\hline NANOG & 100 & 0.05 & 0.01 & 0.02 & A 23 P204640 \\
\hline CDCA4 & 100 & 0.07 & 0.02 & 0.02 & A 23 P205449 \\
\hline ICAI & 100 & 0.13 & 0.01 & 0.02 & A.23 P215419 \\
\hline & & & & & index (fold) \\
\hline
\end{tabular}

Figure 3. hNSC-specific transcriptome. Representative genes were selected with at least 50-fold changes in expression in the hESC-derived hNESs and ReNcell CX cells, compared with the undifferentiated hESCs. The ratios are color-coded, as indicated by the color index bar. hESCs, human embryonic stem cells; hNESs, human neuroectodermal spheres; hEBs, human embryonic bodies; ReN, ReNcell CX cells. 
A

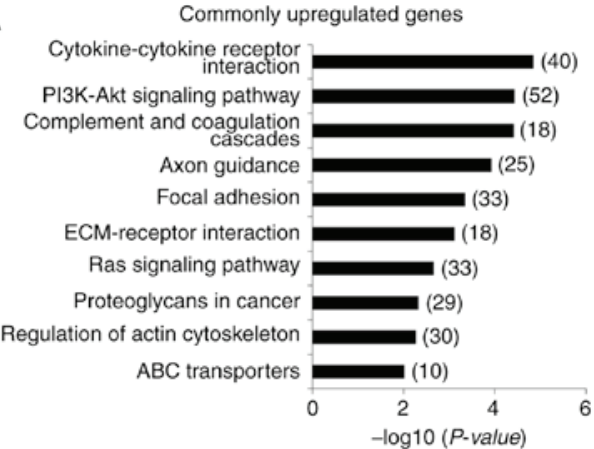

B

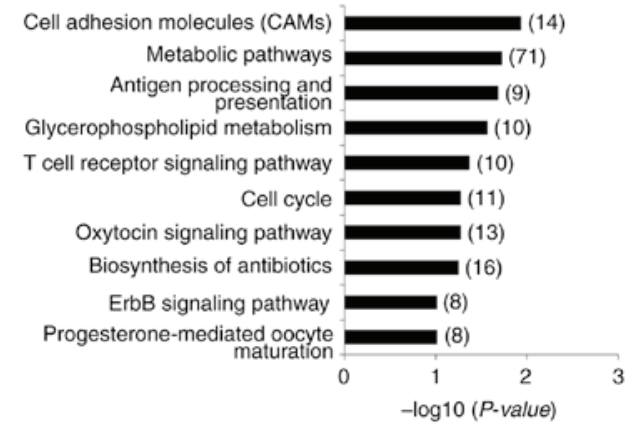

C Annotation cluster/

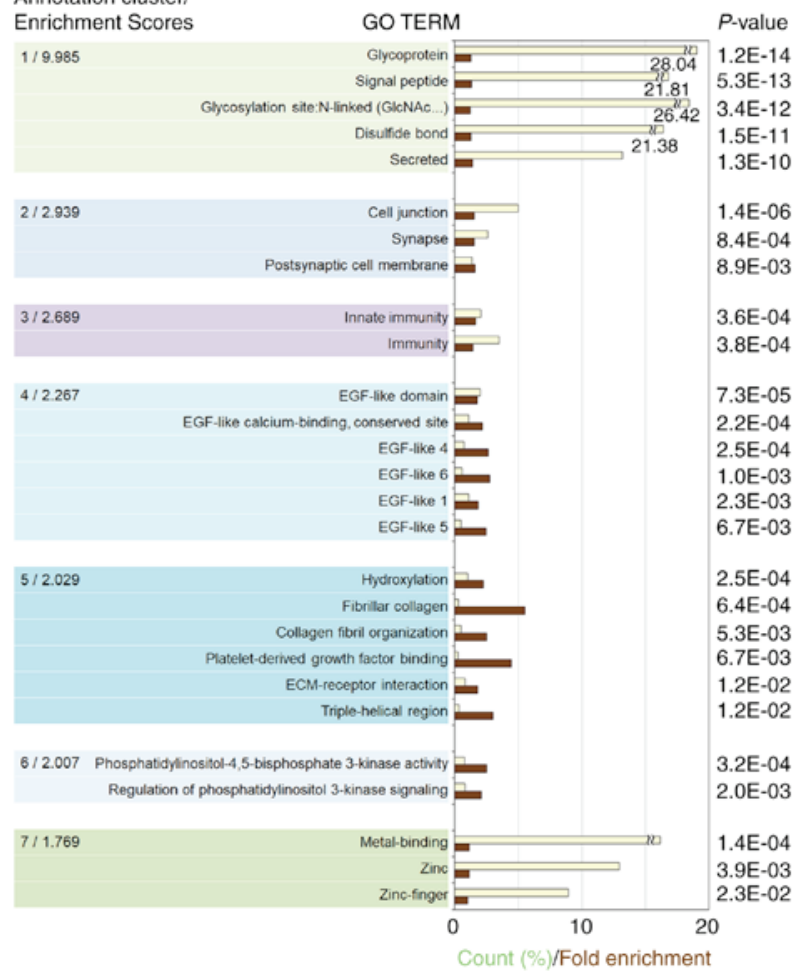

Figure 4. Integrative and comparative analyses of commonly regulated genes in hESC-derived NSCs and ReNcell CX cells. Pathway analysis of commonly (A) upregulated and (B) downregulated genes in hESC-derived hNESs and ReNcell CX cells by Kyoto Encyclopaedia of Genes and Genomes pathway analysis. (C) Functional annotation clustering analysis of commonly regulated genes in hESC-derived hNESs and ReNcell CX cells. The seven most enriched clusters are shown with representative examples of their GO terms and enrichment scores in the colored boxes. The bars show the GO term enrichment (brown) and the number of included proteins relative to the total number of proteins (\% count; in yellow). The P-values are shown on the far right. hESCs, human embryonic stem cells; hNESs, human neuroectodermal spheres; ReN, ReNcell CX cells; GO, Gene Ontology.

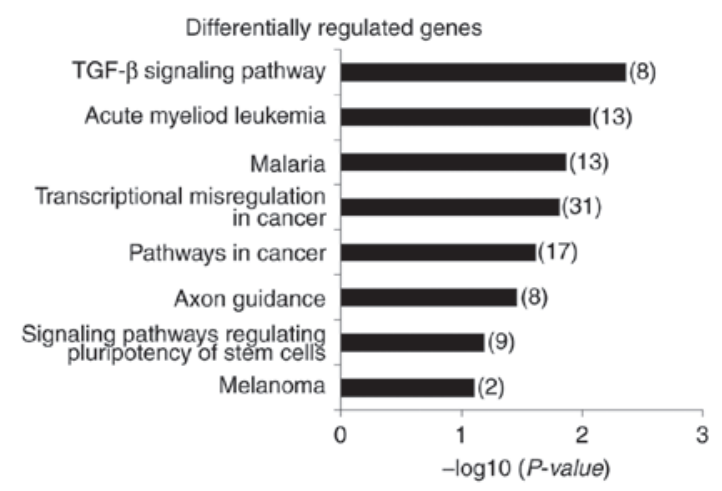

Figure 5. Pathway analysis of the differentially expressed genes between hESC-derived hNESs and ReNcell CX cells. Kyoto Encyclopaedia of Genes and Genomes pathway analysis was performed to analyze the differentially expressed genes. The majority of the over represented pathways were identified as cancer-related pathways. hESCs, human embryonic stem cells; hNESs, human neuroectodermal spheres; ReN, ReNcell CX cells. myeloid leukemia, malaria, transcriptional misregulation in cancer, pathways in cancer, axon guidance, signaling pathways regulating pluripotency of stem cells, and melanoma (Fig. 5). The majority of the overrepresented pathways were identified as cancer-related pathways; this may be due to the oncogenic c-MYC having been functionally linked to cancer-related pathways $(28,29)$.

Neuronal differentiation of hNESs derived from hESCs and ReNcell CX cells in vitro. To further characterize hNESs derived from hESCs molecularly, semi-quantitative RT-PCR analysis was performed for the expression of NSC markers. Transcripts of ESC markers, including OCT4 and NANOG, were decreased during differentiation (Fig. 6A). The levels of molecular markers for NSCs, including superoxide dismutase (SOX)1, SOX2, SOX3, musashi-1 (MSI1), TUJ1 and NESTIN, were increased in the hESC-derived hNESs (Fig. 6A). Dissociated 
A

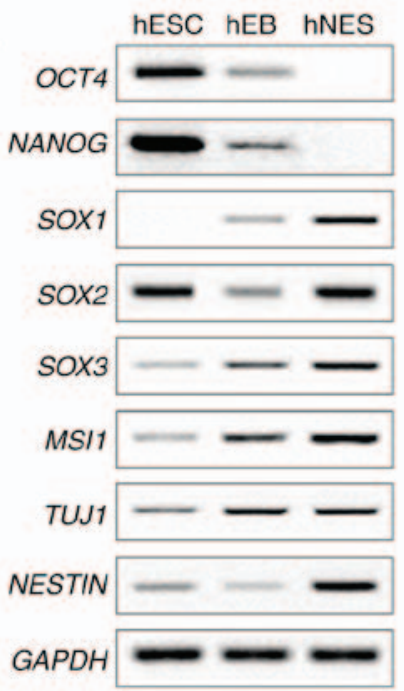

C

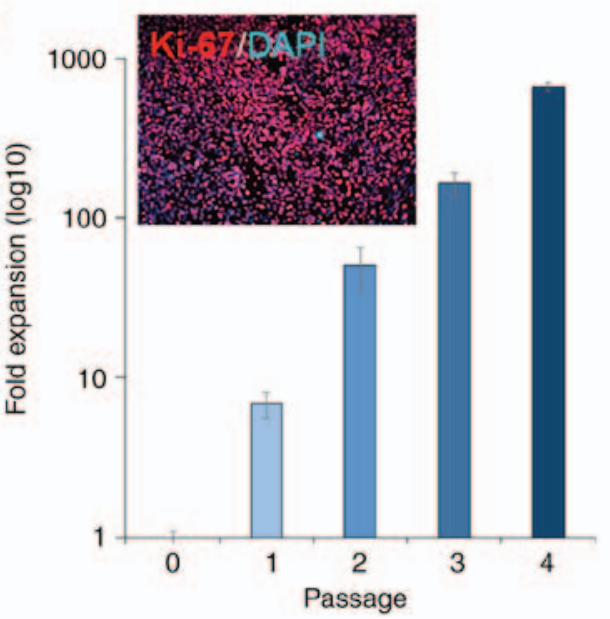

B

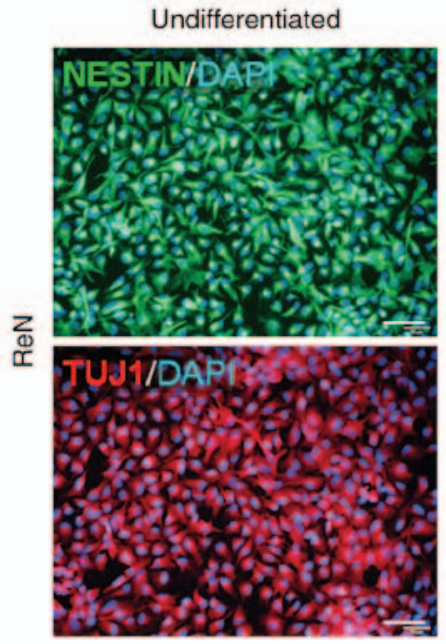

Undifferentiated

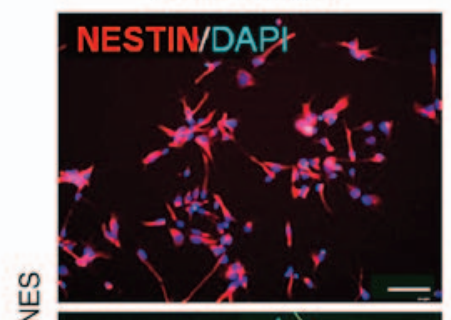

空

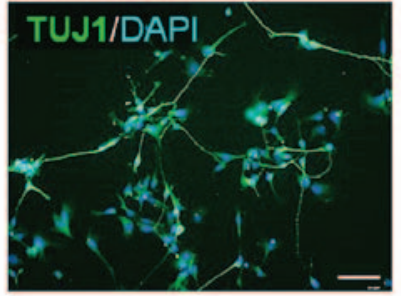

Differentiated

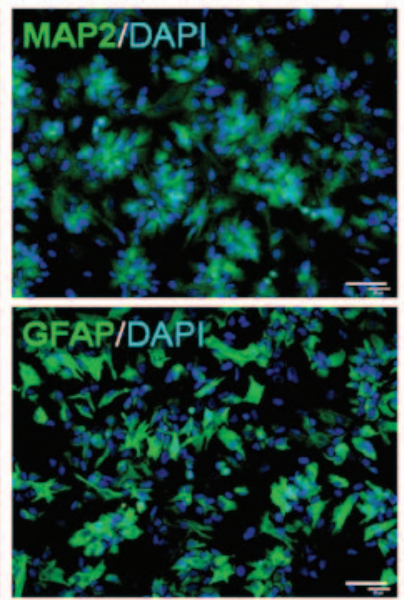

Differentiated
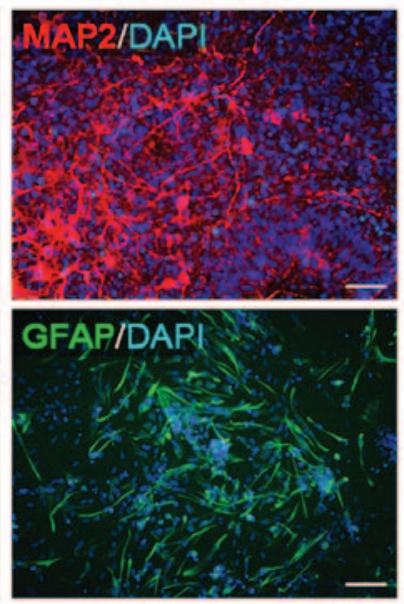

Figure 6. Neuronal differentiation potential of hESC-derived NSCs and ReNcell CX cells. (A) Semi-quantitative reverse transcription-polymerase chain reaction analysis of NSC markers during hNES differentiation. (B) Neuronal differentiation capacity of hESC-derived hNESs and ReNcell CX cells under differentiation conditions. Immunostaining of undifferentiated hNSCs, including hNESs and ReNcell CX cells, with the NSC marker nestin and the neuronal marker TUJ1, and differentiated hNSCs with the mature neuronal marker, MAP2, and the mature glial cell marker, GFAP. Scale bar=200 $\mu \mathrm{m}$. (C) Expansion rate and representative images of Ki-67 staining of hESC-derived hNESs. Fold-expansion was compared with hNESs at passage 0 . hNESs contain Ki-67-positive proliferating NSCs. hESCs, human embryonic stem cells; hNESs, human neuroectodermal spheres; ReN, ReNcell CX cells; hEB, human embryonic body; NSC, neural stem cell; MAP2, microtubule-associated protein 2; GFAP, glial fibrillary acidic protein; TUJ1, neuron-specific class III $\beta$-tubulin; OCT4, octamer-binding protein 4; SOX, superoxide dismutase; MSI1, musashi-1.

hNES cells and ReNcell CX cells were also immunostained for NSC markers, including NESTIN and TUJ1 (Fig. 6B). To functionally characterize neuronal differentiation capacity, hNESs and ReNcell CX cells were differentiated following the withdrawal of growth factors. Following 15 days of differentiation, MAP2-positive neuronal cells and GFAP-positive glial cells were observed in the differentiated hNESs and ReNcell CX cells (Fig. 6B). Of note, the hESC-derived hNESs continued to expand over five passages without losing any of their features. It was possible to scale-up hNES production by $\sim 662$-fold in terms of cell number (mean values) following five passages, with the majority of cells undergoing active proliferation, as indicated by Ki-67 labeling at passage 5 (Fig. 6C).

The aim of the present study was to characterize hNSCs and to select a more suitable hNSC model for developing human-based platforms for applications in various neurological fields. The hNSC-specific transcriptome data from hESC-derived hNESs and ReNcell CX hNSCs were described.
Global gene expression profiling enables a systems-based analysis of the biological processes through GO and pathway enrichment analyses, and of genes driving differentiation into hNSCs. The comparative analysis of the global gene expression showed that the hESC-derived hNESs were similar to the ReNcell CX hNSCs, as shown by PCA and hierarchical sample clustering. As described above, the hNESs derived from hESCs in the simple protocol exhibited differentiation potential, based on the specific terminal differentiation markers of neuronal and glial cells that were detected. The hNESs showed stable proliferation and were expanded for at least five passages without loss of NSC characteristics. These results indicated that hESC-derived hNESs may be used as a relevant hNSC model, similar to ReNcell CX cells, in several neurological research fields.

hNSC differentiation protocols remain inefficient, with poor yields of terminal differentiation and maturation into specific neuronal cell types. However, increased understanding 
of the mechanisms underlying hESC-based NSC differentiation through fine-tuning protocols for the efficient derivation, long-term maintenance and neuronal cell type-specific differentiation of hNSCs may provide novel insights into human neurodevelopment and the process of NSC fate specification.

\section{Acknowledgements}

This study was supported by the National Research Foundation of Korea grant funded by the Ministry of Science, ICT and Future Planning (grant nos. 2016R1A2B4013501 and NRF-2016M3A9C4953144) and a grant from the KRIBB Research Initiative Program. The funders had no involvement in study design, data collection or analysis, decision to publish or preparation of the manuscript.

\section{References}

1. Oh JH, Son MY, Choi MS, Kim S, Choi AY, Lee HA, Kim KS Kim J, Song CW and Yoon S: Integrative analysis of genes and miRNA alterations in human embryonic stem cells-derived neural cells after exposure to silver nanoparticles. Toxicol Appl Pharmacol 299: 8-23, 2016.

2. Son MY, Sim H, Son YS, Jung KB, Lee MO, Oh JH, Chung SK, Jung CR and Kim J: Distinctive genomic signature of neural and intestinal organoids from familial Parkinson's disease patient-derived induced pluripotent stem cells. Neuropathol Appl Neurobiol 43: 584-603, 2017.

3. Otsu M, Nakayama T and Inoue N: Pluripotent stem cell-derived neural stem cells: From basic research to applications. World J Stem Cells 6: 651-657, 2014.

4. Zhao X, Yang Z, Liang G, Wu Z, Peng Y, Joseph DJ, Inan S and Wei H: Dual effects of isoflurane on proliferation, differentiation, and survival in human neuroprogenitor cells. Anesthesiology 118: 537-549, 2013

5. Díaz-Coránguez M, Segovia J, López-Ornelas A, Puerta-Guardo H, Ludert J, Chávez B, Meraz-Cruz N and González-Mariscal L: Transmigration of neural stem cells across the blood brain barrier induced by glioma cells. PLoS One 8: e60655, 2013.

6. Hovakimyan M, Müller J, Wree A, Ortinau S, Rolfs A and Schmitt O: Survival of transplanted human neural stem cell line (ReNcell VM) into the rat brain with and without immunosuppression. Ann Anat 194: 429-435, 2012.

7. Choi SH, Kim YH, Quinti L, Tanzi RE and Kim DY: 3D culture models of Alzheimer's disease: A road map to a 'cure-in-a-dish' Mol Neurodegener 11: 75, 2016.

8. Kim YH, Choi SH, D'Avanzo C, Hebisch M, Sliwinski C, Bylykbashi E, Washicosky KJ, Klee JB, Brüstle O, Tanzi RE and Kim DY: A 3D human neural cell culture system for modeling Alzheimer's disease. Nat Protoc 10: 985-1006, 2015.

9. Woo SM, Kim J, Han HW, Chae JI, Son MY, Cho S, Chung HM, Han YM and Kang YK: Notch signaling is required for maintaining stem-cell features of neuroprogenitor cells derived from human embryonic stem cells. BMC Neurosci 10: 97, 2009.

10. Breunig JJ, Haydar TF and Rakic P: Neural stem cells: Historical perspective and future prospects. Neuron 70: 614-625, 2011.

11. Son MY, Kwak JE, Kim YD and Cho YS: Proteomic and network analysis of proteins regulated by REX1 in human embryonic stem cells. Proteomics 15: 2220-2229, 2015.

12. Chambers SM, Fasano CA, Papapetrou EP, Tomishima M, Sadelain M and Studer L: Highly efficient neural conversion of human ES and iPS cells by dual inhibition of SMAD signaling. Nat Biotechnol 27: 275-280, 2009.
13. Dhara SK and Stice SL: Neural differentiation of human embryonic stem cells. J Cell Biochem 105: 633-640, 2008.

14. Son MY, Lee MO, Jeon H, Seol B, Kim JH, Chang JS and Cho YS: Generation and characterization of integration-free induced pluripotent stem cells from patients with autoimmune disease. Exp Mol Med 48: e232, 2016.

15. Jung KB, Son YS, Lee H, Jung CR, Kim J and Son MY: Transcriptome dynamics of human pluripotent stem cell-derived contracting cardiomyocytes using an embryoid body model with fetal bovine serum. Mol Biosyst 13: 1565-1574, 2017.

16. Kim DS, Ryu JW, Son MY, Oh JH, Chung KS, Lee S, Lee JJ, Ahn JH, Min JS, Ahn J, et al: A Liver-specific Gene Expression panel predicts the differentiation status of in vitro hepatocyte models. Hepatology 66: 1662-1674, 2017.

17. Son MY, Kwak JE, Seol B, Lee DY, Jeon H and Cho YS: A novel human model of the neurodegenerative disease GM1 gangliosidosis using induced pluripotent stem cells demonstrates inflammasome activation. J Pathol 237: 98-110, 2015.

18. Son MY, Kim HJ, Kim MJ and Cho YS: Physical passaging of embryoid bodies generated from human pluripotent stem cells. PLoS One 6: e19134, 2011.

19. Nakagawa M, Takizawa N, Narita M, Ichisaka T and Yamanaka S: Promotion of direct reprogramming by transformation-deficient Myc. Proc Natl Acad Sci USA 107: 14152-14157, 2010.

20. Kwak JE, Son MY, Son YS, Son MJ and Cho YS: Biochemical and molecular characterization of novel mutations in GLB1 and NEU1 in patient cells with lysosomal storage disorders. Biochem Biophys Res Commun 457: 554-560, 2015.

21. Jung KB, Lee H, Son YS, Lee JH, Cho HS, Lee MO, Oh JH, Lee J, Kim S, Jung CR, et al: In vitro and in vivo imaging and tracking of intestinal organoids from human-induced pluripotent stem cells. FASEB J: Aug 29, 2017 (Epub ahead of print). doi: 10.1096/fj.201700504R

22. Livak KJ and Schmittgen TD: Analysis of relative gene expression data using real-time quantitative PCR and the 2(-Delta Delta C(T)) method. Methods 25: 402-408, 2001.

23. Son MY, Kim YD, Seol B, Lee MO, Na HJ, Yoo B, Chang JS and Cho YS: Biomarker discovery by modeling behcet's disease with patient-specific human induced pluripotent stem cells. Stem Cells Dev 26: 133-145, 2017.

24. Donato R, Miljan EA, Hines SJ, Aouabdi S, Pollock K, Patel S, Edwards FA and Sinden JD: Differential development of neuronal physiological responsiveness in two human neural stem cell lines. BMC Neurosci 8: 36, 2007.

25. Li Z, Oganesyan D, Mooney R, Rong X, Christensen MJ, Shahmanyan D, Perrigue PM, Benetatos J, Tsaturyan L, Aramburo S, et al: L-MYC expression maintains self-renewal and prolongs multipotency of primary human neural stem cells. Stem Cell Reports 7: 483-495, 2016.

26. Li X, Xu J, Bai Y, Wang X, Dai X, Liu Y, Zhang J, Zou J, Shen L and $\mathrm{Li} \mathrm{L}$ : Isolation and characterization of neural stem cells from human fetal striatum. Biochem Biophys Res Commun 326: 425-434, 2005.

27. Huang da W, Sherman BT and Lempicki RA: Systematic and integrative analysis of large gene lists using DAVID bioinformatics resources. Nat Protoc 4: 44-57, 2009.

28. Mazan-Mamczarz K, Hagner PR, Dai B, Wood WH, Zhang Y, Becker KG, Liu Z and Gartenhaus RB: Identification of transformation-related pathways in a breast epithelial cell model using a ribonomics approach. Cancer Res 68: 7730-7735, 2008.

29. Miller DM, Thomas SD, Islam A, Muench D and Sedoris K: c-Myc and cancer metabolism. Clin Cancer Res 18: 5546-5553, 2012 . 